\title{
Seroprevalence and risk factors of Chlamydia abortus infection in free-ranging white yaks in China
}

\author{
Si-Yuan Qin ${ }^{1,2}$, Si-Yang Huang ${ }^{2}$, Ming-Yang Yin ${ }^{2,3}$, Qi-Dong Tann ${ }^{2,4}$, Guang-Xue Liư ${ }^{2}$ Dong-Hui Zhou², \\ Xing-Quan Zhu ${ }^{1,2}$, Ji-Zhang Zhou ${ }^{2^{*}}$ and Ai-Dong Qian ${ }^{1 *}$
}

\begin{abstract}
Background: Chlamydia is gram-negative obligate bacteria which causes a wide variety of diseases in humans and animals. To date, there are a few reports about the seroprevalence of Chlamydia and the risk factors associated with Chlamydia infection in yaks in the world. In this study, 974 blood samples were collected from white yaks (Bos grunniens) in Tianzhu Tibetan Autonomous County, Gansu province, northwest China from June 2013 to April 2014.

Results: Antibodies against Chlamydia abortus were examined by the indirect hemagglutination (IHA) test, and 158 of 974 (16.22\%) white yaks were seropositive for C. abortus antibodies at the cut-off of 1:16. The risk factors associated with seroprevalence were evaluated by a multivariate logistic regression analysis. Region, gender and age of white yak were left out of the final model, due to its insignificance in the logistic regression analysis $(P>0.05)$. However, season was considered as a major risk factor associated with C. abortus infection in white yaks.
\end{abstract}

Conclusions: To our knowledge, this is the first survey of C. abortus seroprevalence in white yaks in China, which extends the host range for C. abortus and has important implications for public health and the local Tibetan economy.

Keywords: Chlamydia abortus, White yaks, Seroprevalence, Tibetans, China

\section{Background}

Chlamydia spp. are obligate intracellular bacteria which are the etiological agents of chlamydiosis and cause a broad spectrum of diseases in animals and humans [1,2]. Chlamydia are among the main pathogens for infection of the eye and the urogenital and respiratory tracts in humans, and chronic and repeated infection may result in severe irreversible damage such as blindness (trachoma) and tubal infertility [1].

Chlamydiaceae has a single genus Chlamydia that currently comprises twelve species [3-6]. Previously studies indicated that some of them can infect cattle, including Chlamydia pecorum, C. abortus, C. psittaci, and C. suis [7]. C. abortus is one of the most important pathogens

\footnotetext{
*Correspondence: zhoujizhang@caas.cn; qianaidong0115@163.com ${ }^{2}$ State Key Laboratory of Veterinary Etiological Biology, Lanzhou Veterinary Research Institute, Chinese Academy of Agricultural Sciences, Lanzhou, Gansu Province 730046, PR China

${ }^{1}$ College of Animal Science and Technology, Jilin Agricultural University, Changchun, Jilin Province 130118, PR China

Full list of author information is available at the end of the article
}

which lead to reproductive failure in yaks, especially in arctic-alpine areas in China and other countries. Furthermore, pregnant women can be infected through contacting with animals infected with $C$. abortus, so it is also a zoonotic pathogen [2]. Recently, C. abortus infection in cattle has been reported in many countries such as UK, Sweden, Germany, Jordan, Ireland, Switzerland, Slovak Republic and Poland [8-15], as well as China [16,17].

White yak is a semi-natural and rare breed of cattle in the world which only lives in Tianzhu Tibetan Autonomous County, Gansu province, northwest China with a condition of alpine hypoxia and low temperature [18]. Milk and meat of white yaks are the popular delicacy for local Tibetan people and other residents in Gansu Province. However, limited data about C. abortus infection was available in yaks all over the world $[16,19]$, and no information on the prevalence of $C$. abortus in white yaks is available. The objective of the present survey was to investigate the seroprevalence and risk factors including region, gender, age and season of C. abortus infection in white yaks 
in Tianzhu Tibetan Autonomous County, northwestern China. The results may provide essential information to prevent and control C. abortus infection in white yaks.

\section{Methods}

\section{Ethics statement}

This study was approved by the Animal Ethics Committee of Lanzhou Veterinary Research Institute, Chinese Academy of Agricultural Sciences (Approval No. LVRIAEC2013-010). The white yaks from which the serum samples were collected were handled in accordance with good animal practices required by the Animal Ethics Procedures and Guidelines of the People's Republic of China.

\section{The investigation site}

The present study was carried out in Xidatan village and Zhuaxixiulong village in Tianzhu Tibetan Autonomous County. The county has an area of 7,149 square kilometers, and the vast majority of its land is more than 3,000 meters above sea level. The average temperature of this region is $-8^{\circ} \mathrm{C}$ to $4^{\circ} \mathrm{C}$. In this mountainous area, the free-range white yaks feed on local weeds and brook, which only live in Tianzhu Tibetan Autonomous County in China.

\section{Serum samples}

A total of 974 blood samples were collected from white yaks in Tianzhu Tibetan Autonomous County of Gansu province between June 2013 and April 2014. The white yaks were randomly selected, blood samples of which were transported to the laboratory, kept at room temperature for $2 \mathrm{~h}$ and centrifuged at $3000 \mathrm{~g}$ for $10 \mathrm{~min}$ to separate clear serum. The serum samples were stored at $-20^{\circ} \mathrm{C}$ until further analysis.

\section{Serological examination}

A commercially available Indirect Hemagglutination Assay (IHA) kit (Lanzhou Veterinary Research Institute, Chinese Academy of Agricultural Sciences, Lanzhou, China) was purchased to test antibodies to C. abortus and it was carried out according to the manufacturer's instructions as described previously [16]. The Ministry of Agriculture of the People's Republic of China (NY/T 562-2002) has confirmed the sensitivity and specificity values for the testing kit used in this study. In brief, serum samples were added to 96-well V-bottomed polystyrene plates, which were diluted 4-fold serially beginning with 1:4 to 1:1,024. Each test was performed with positive, negative and blank controls, and serum samples which had positive reaction at dilutions of 1:16 or higher dilutions were considered positive for C. abortus antibodies.

\section{Risk factors}

Information about age, gender, sampling season and geographical origin of yaks were collected from the owners of white yaks. The ages of sampled yaks were divided into four categories: $\mathrm{yr} \leq 1,1<\mathrm{yr} \leq 2,2<\mathrm{yr} \leq 3$ and $\mathrm{yr}>3$. The sampling seasons were distributed in spring (March to May), summer (June to August), autumn (September to November) and winter (December to February). Two regions and two genders of yaks were also analyzed in this study.

\section{Statistical analyses}

Variables associated with $C$. abortus infection among white yaks of different geographical origins, gender, seasons and age groups were analyzed in a multivariable logistic regression model, probability $(P)$ value $<0.05$ was considered as statistically significant between factors and prevalence. Odds-ratios (OR) with $95 \%$ confidence intervals based on likelihood ratio statistics are reported. All statistical analyses were performed using the PASW Statistics 18.0 (SPSS Inc., IBM Corporation, Somers, NY).

\section{Results}

In this study, serum samples were collected from a total of 974 white yaks (73 from Zhuaxixiulong village and 901 from Xidatan village) in Tianzhu Tibetan Autonomous County, Gansu province, northwest China. 158 out of 974 serum samples (16.22\%) were seropositive for C. abortus by IHA test at the cutoff dilution of 1:16 (Table 1). The seroprevalence was $28.77 \%$ (21/73) in Zhuaxixiulong village and $15.21 \%$ (137/901) in Xidatan village. Seroprevalence of $C$. abortus infection in male and female white yaks were $17.30 \%(50 / 289)$ and $15.78 \%(108 / 685)$, respectively (Table 1). The ages of the examined white yaks varied from 0 year to 3 years or greater and the seroprevalence ranged from $12.17 \%$ to $20.57 \%$. The seroprevalence in spring, summer, autumn and winter were $10.91 \%, 24.91 \%$, $11.26 \%$ and $16.00 \%$, respectively (Table 1 ). The antibodies titers were diverse in different regions, genders, ages and seasons with the most frequent level of 1:16 in 93 samples (58.86\%), followed by 1:32 in 43 samples $(27.22 \%), 1: 64$ in 16 samples (10.13\%), 1:128 in 4 samples (2.53\%) and 1:256 in 2 samples (1.27\%) (Table 2).

According to forward stepwise logistic regression, region, age and gender of white yak were not significant in the logistic regression analysis $(P>0.05)$ and left out of the final model (Hosmer and Lemeshow goodness of fit test $P=1.00$ ). Only one factor, season of collecting samples was considered as main risk factor to influence the seroprevalence significantly (Table 3 ).

\section{Discussion}

The epidemiological information regarding the prevalence of C. abortus in yaks are limited in the world, including China. There were described cases in yaks in India and China but no information on the prevalence of C. abortus 
Table 1 Seroprevalence of Chlamydia abortus infection in white yaks associated with different factors in Tianzhu Tibetan Autonomous County, northwest China by indirect hemagglutination assay (IHA)

\begin{tabular}{|c|c|c|c|c|}
\hline Biometric data & Category & No. tested & No. positive & Prevalence (\%) \\
\hline \multirow[t]{2}{*}{ Region } & Zhuaxixiulong & 73 & 21 & 28.77 \\
\hline & Xidatan & 901 & 137 & 15.21 \\
\hline \multirow[t]{2}{*}{ Sex } & Male & 289 & 50 & 17.30 \\
\hline & Female & 685 & 108 & 15.78 \\
\hline \multirow[t]{4}{*}{ Season } & Spring & 220 & 24 & 10.91 \\
\hline & Summer & 273 & 68 & 24.91 \\
\hline & Autumn & 231 & 26 & 11.26 \\
\hline & Winter & 250 & 40 & 16.00 \\
\hline \multirow[t]{4}{*}{ Age (yr) } & $0<y r \leq 1$ & 115 & 14 & 12.17 \\
\hline & $1<y r \leq 2$ & 180 & 27 & 15.00 \\
\hline & $2<y r \leq 3$ & 175 & 36 & 20.57 \\
\hline & $>3$ & 504 & 81 & 16.07 \\
\hline Total & & 974 & 158 & 16.22 \\
\hline
\end{tabular}

in white yaks. The present study, therefore, aimed at estimating $C$. abortus seroprevalence in white yaks. The results revealed the presence of $C$. abortus in $16.22 \%$ of white yaks in the present study, which was lower than the value of $35 \%$ in yaks in India [19] and similar to the $17.66 \%$ seroprevalence in black yaks in Qinghai province, China [16]. The differences in the seroprevalence of $C$. abortus exposure in yaks in different regions could be related to differences in ecological and geographical factors including temperature, rainfall or landscape differences. In addition, sanitation and husbandry practices in yak production could be other reasons for the variation of prevalence.
The significant different seroprevalence observed may also be caused by the different methods, including antigen ELISA, IHA, conventional or multiplex PCR. However, IHA is considered as a simple, safe, and reliable means of testing $C$. abortus antibodies, which has been employed in previous serological investigations $[16,20]$. The sensitivity and specificity of the testing IHA kit have been validated by the Ministry of Agriculture of the People's Republic of China, which demonstrates that the IHA is not only more efficient than the CFT but is also more inexpensive than the ELISA [21]. Therefore, it may be the most appropriate commercially available kit for detecting C. abortus infection in white yaks.

Table 2 Antibody titers of Chlamydia abortus infection in white yaks in Tianzhu Tibetan Autonomous County, China determined by indirect haemagglutination (IHA) test

\begin{tabular}{|c|c|c|c|c|c|c|c|c|c|}
\hline \multirow[t]{2}{*}{ Biometric data } & \multirow[t]{2}{*}{ Category } & \multicolumn{5}{|c|}{ Antibody titers } & \multirow[t]{2}{*}{ No. positive } & \multirow[t]{2}{*}{ No. tested } & \multirow[t]{2}{*}{ Prevalence (\%) } \\
\hline & & $1: 16$ & $1: 32$ & $1: 64$ & $1: 128$ & $1: 256$ & & & \\
\hline \multirow[t]{2}{*}{ Region } & Zhuaxixiulong & 10 & 7 & 2 & 1 & 1 & 21 & 73 & 28.77 \\
\hline & Xidatan & 83 & 36 & 14 & 3 & 1 & 137 & 901 & 15.21 \\
\hline \multirow[t]{2}{*}{ Sex } & Male & 24 & 18 & 7 & 1 & 0 & 50 & 289 & 17.30 \\
\hline & Female & 69 & 25 & 9 & 3 & 2 & 108 & 685 & 15.78 \\
\hline \multirow[t]{4}{*}{ Age (years) } & $\leq 1$ & 11 & 1 & 2 & 0 & 0 & 14 & 115 & 12.17 \\
\hline & $1<y r \leq 2$ & 22 & 2 & 3 & 0 & 0 & 27 & 180 & 15.00 \\
\hline & $2<y r \leq 3$ & 15 & 15 & 5 & 1 & 0 & 36 & 175 & 20.57 \\
\hline & $y r>3$ & 45 & 25 & 6 & 3 & 2 & 81 & 504 & 16.07 \\
\hline \multirow[t]{5}{*}{ Season } & Spring & 18 & 3 & 3 & 0 & 0 & 24 & 220 & 10.91 \\
\hline & Autumn & 17 & 8 & 1 & 0 & 0 & 26 & 231 & 11.26 \\
\hline & Winter & 27 & 6 & 6 & 0 & 1 & 40 & 250 & 16.00 \\
\hline & Summer & 31 & 26 & 6 & 4 & 1 & 68 & 273 & 24.91 \\
\hline & Total & 93 & 43 & 16 & 4 & 2 & 158 & 974 & 16.22 \\
\hline
\end{tabular}


Table 3 Odds ratios for seasons of white yak are taken as risk factors for Chlamydia abortus seroprevalence in white yaks

\begin{tabular}{lllllll}
\hline Factor & Category & No. tested & No. positive & Prevalence (\%) & OR (95\% CI) & Reference \\
\hline Season & Spring & 220 & 24 & 10.91 & 11.26 & $1.04(0.58-1.87)$ \\
& Autumn & 231 & 26 & 16.00 & $1.56(0.90-2.68)$ & 0.907 \\
& Winter & 250 & 68 & 24.91 & $2.71(1.64-4.49)$ & 0.110 \\
& Summer & 273 & 40 & 0.001 \\
\hline
\end{tabular}

In the present study, statistical analysis showed that there was no significant difference in seroprevalence between male and female yaks $(P>0.05)$, suggesting that gender may not be a crucial factor for $C$. abortus infection in white yaks. The result is consistent with the studies of Chen et al. [16] in which they reported no association between sex and $C$. abortus prevalence in black yaks in Qinghai province. The highest prevalence $(20.57 \%)$ was found in the $2<$ years $\leq 3$ age group, while the lowest prevalence was found in younger white yaks ( $12.17 \%$ in $0<$ years $\leq 1$ age group). Age of white yaks as a continuous variable was analyzed in the logistic regression model, the results showed that there is no significant difference in $C$. abortus seroprevalence in white yaks of different ages $(P>0.05)$, which is in agreement with a previous studies [16]. In addition, the geographical origin of white yaks is also not a risk factor associated with $C$. abortus seroprevalence through logistic regression analysis $(P>0.05)$, although the seroprevalence in Zhuaxixiulong village was much higher than that in Xidatan village. The number of yak samples collected from Zhuaxixiulong is much smaller than that in Xidatan, on account of small white yak population in Zhuaxixiulong village, which may have a significant effect upon the statistical tests used.

This study revealed that season is a significant risk factor associated with C. abortus prevalence due to different climates in different seasons, including diverse temperature, precipitation and humidity. The highest seroprevalence was in summer followed by winter, autumn and spring, and the higher seroprevalence in summer may be related to producing antibody following abortion. Abortion usually occurred in spring resulting in generating much antibody for resistance to C. abortus and the antibody titers would reach its highest peak in summer. After abortion, the rubbish and excretion of abortion may be contacted by other healthy white yaks, which increased the number of infected white yak in summer. Therefore, it is necessary to take effective measures to prevent the spread of $C$. abortus during reproductive seasons.

Logistic regression analysis showed that white yaks in summer $(24.91 \%)$ had a 2.7 times higher risk of being seropositive compared to white yaks in spring (10.91\%) $(\mathrm{OR}=2.71,95 \% \mathrm{CI}=1.64-4.49, P<0.001)$, although no seasonal differences were found among other two seasons compared to spring $(P>0.05)$. Such significant season differences in prevalence may be associated with changes in climate conditions. The warm temperature and appropriate precipitation in summer are predicted to elevate the survival of Chlamydia, leading to increased transmission to white yaks in this area.

IHA is considered as a simple, fast and inexpensive method in the serological survey of Chlamydia infection which has been used to detect some kinds of antibodies against Chlamydia in several animal species [20,22-24]. 974 white yak samples were collected in Tianzhu Tibetan Autonomous County,where there are only approximately 49,400 white yaks [25]. Although survey results may not reflect the status of $C$. abortus infection in all the white yaks, our results could provide useful information for the effective prevention and control of chlamydiosis in white yaks.

\section{Conclusions}

Results of the epidemiological study revealed a high C. abortus seroprevalence in white yaks from Tianzhu Tibetan Autonomous County, which can cause economic losses to the local cattle industry, and also pose a potential threat to local Tibetans health in this area. This report extends the host range for C. abortus, and provides basal information to prevent and control C. abortus infection in white yaks.

\section{Abbreviations}

IHA: Indirect hemagglutination; C. abortus: Chlamydia abortus; C. pecorum: Chlamydia pecorum; C. psittaci: Chlamydia psittaci; C. suis: Chlamydia suis; OR: Odds-ratios; P: Probability; ELISA: Enzyme-linked immunosorbent assay; PCR: Polymerase chain reaction; CFT: Complement fixation test; $\mathrm{Cl}$ : Confidence interval.

\section{Competing interests}

The authors declare that they have no competing interests.

\section{Authors' contributions}

ADQ and JZZ conceived and designed the study, and critically revised the manuscript. SYQ, SYH, MYY, QDT, GXL and DHZ collected the sample, performed the experiments, analyzed the data and drafted the manuscript. XQZ helped in study design, study implementation and manuscript revision. All authors read and approved the final manuscript.

\section{Acknowledgments}

Project support was provided, in part, by the National Natural Science Foundation of China (Grant No. 31272566), the Science Fund for Creative Research Groups of Gansu Province (Grant No. 1210RJIA006) and the Special Fund for Agro-scientific Research in the Public Interest (Grant No. 201303037). 


\section{Author details}

${ }^{1}$ College of Animal Science and Technology, Jilin Agricultural University, Changchun, Jilin Province 130118, PR China. ${ }^{2}$ State Key Laboratory of Veterinary Etiological Biology, Lanzhou Veterinary Research Institute, Chinese Academy of Agricultural Sciences, Lanzhou, Gansu Province 730046, PR China. ${ }^{3}$ College of Veterinary Medicine, Hunan Agricultural University, Changsha, Hunan Province 410128, PR China. ${ }^{4}$ College of Animal Science and Technology, Anhui Agricultural University, Hefei, Anhui Province 230000, PR China.

Received: 5 October 2014 Accepted: 12 January 2015

Published online: 20 January 2015

\section{References}

1. Rohde G, Straube E, Essig A, Reinhold P, Sachse K. Chlamydial zoonoses. Dtsch Arztebl Int. 2010;107:174-80.

2. Schautteet $K$, Vanrompay D. Chlamydiaceae infections in pig. Vet Res. 2011:42:1-10.

3. Longbottom D, Coulter L. Animal chlamydioses and zoonotic implications. J Comp Pathol. 2003;128:217-44.

4. Stephens RS, Myers G, Eppinger M, Bavoil PM. Divergence without difference: phylogenetics and taxonomy of Chlamydia resolved. FEMS Immunol Med Microbiol. 2009;55:115-9.

5. Sachse K, Laroucau K, Riege K, Wehner S, Dilcher M, Creasy HH, et al. Evidence for the existence of two new members of the family Chlamydiaceae and proposal of Chlamydia avium sp. nov. and Chlamydia gallinacea sp. nov. Syst Appl Microbiol. 2014;37:79-88.

6. Vorimore F, Hsia RC, Huot-Creasy H, Bastian S, Deruyter L, Passet A, et al. Isolation of a New Chlamydia species from the Feral Sacred Ibis (Threskiornis aethiopicus): Chlamydia ibidis. PLoS One. 2013;8:e74823.

7. Reinhold P, Sachse K, Kaltenboeck B. Chlamydiaceae in cattle: commensals, trigger organisms, or pathogens? Vet J. 2011;189:257-67.

8. Blumer S, Greub G, Waldvogel A, Hassig M, Thoma R, Tschuor A, et al. Waddlia, Parachlamydia and Chlamydiaceae in bovine abortion. Vet Microbiol. 2011;152:385-93.

9. Karlsson AC, Alenius S, Bjorkman C, Persson Y, Englund S. Investigation of Chlamydiaceae in semen and cauda epididymidis and seroprevalence of Chlamydophila abortus in breeding bulls. Acta Vet Scand. 2010;52:2.

10. Kauffold J, Henning K, Bachmann R, Hotzel H, Melzer F. The prevalence of chlamydiae of bulls from six bull studs in Germany. Anim Reprod Sci. 2007; 102:111-21.

11. Talafha AQ, Ababneh MM, Ababneh MM, Al-Majali AM. Prevalence and risk factors associated with Chlamydophila abortus infection in dairy herds in Jordan. Trop Anim Health Prod. 2012:44:1841-6.

12. Wheelhouse N, Howie F, Gidlow J, Greub G, Dagleish M, Longbottom D. Involvement of Parachlamydia in bovine abortions in Scotland. Vet 2012;193:586-8.

13. Wilson K, Sammin D, Harmeyer S, Nath M, Livingstone M, Longbottom D. Seroprevalence of chlamydial infection in cattle in Ireland. Vet J. 2012;193:583-5.

14. Travnicek M, Kovacova D, Deptula W, Bajova V, Cislakova L, Zubricky P, et al. Serological response of cattle to Chlamydophila abortus in Slovakia in 1996-2000. Pol J Vet Sci. 2002;5:25-7.

15. Szymanska-Czerwinska M, Niemczuk K, Galinska EM. Serological and nested PCR survey to determine the occurrence of chlamydia infections in the Polish cattle population. Ann Agric Environ Med. 2013;20:682-6.

16. Chen Q, Gong X, Zheng F, Cao X, Li Z, Zhou J. Seroprevalence of Chlamydophila abortus infection in yaks (Bos grunniens) in Qinghai, China. Trop Anim Health Prod. 2014;46:503-7.

17. Zhou DH, Zhao FR, Xia HY, Xu MJ, Huang SY, Song HQ, et al. Seroprevalence of chlamydial infection in dairy cattle in Guangzhou, southern China. Ir Vet J. 2013;66:2

18. Qin SY, Zhang XX, Zhao GH, Zhou DH, Yin MY, Zhao Q, et al. First report of Cryptosporidium spp. in white yaks in China. Parasit Vectors. 2014;7:230.

19. Bandyopadhyay S, Sasmal D, Biswas TK, Samanta I, Ghosh MK. Serological evidence of antibodies against Chlamydophila abortus in free-ranging yak (Poephagus grunniens) in Arunachal Pradesh, India. Rev Sci Tech. 2009:28:1051-5.

20. Qin SY, Yin MY, Cong W, Zhou DH, Zhang XX, Zhao Q, et al. Seroprevalence and risk factors of Chlamydia abortus infection in Tibetan sheep in Gansu Province, northwest China. ScientificWorldJournal. 2014;2014:193464.
21. Jiang TT, Yang YS, Meng QY, Fang YL, Han SL. Study on detecting Chlamydia in swine by indirect hemagglutination assay. Hubei J Anim Vet sci. 1983;3:15-21.

22. Cong W, Huang SY, Zhang XX, Zhou DH, Xu MJ, Zhao Q, et al. Chlamydia psittaci exposure in pet birds. J Med Microbiol. 2014;63:578-81.

23. Cong W, Huang SY, Zhang XY, Zhou DH, Xu MJ, Zhao Q, et al. Seroprevalence of Chlamydia psittaci infection in market-sold adult chickens, ducks and pigeons in north-western China. J Med Microbiol. 2013;62:1211-4.

24. Jiang HH, Huang SY, Zhang W, Zhao L, Xu C, Deng S, et al. Seroprevalence of Chlamydia infection in pigs in Jiangxi province, southeastern China. J Med Microbiol. 2013;62:1864-7.

25. Cang M. Study on sustainable development strategies of Tianzhu white yak industry. Acta Ecol Anim Domast. 2008:29:133-5.

\section{Submit your next manuscript to BioMed Central and take full advantage of:}

- Convenient online submission

- Thorough peer review

- No space constraints or color figure charges

- Immediate publication on acceptance

- Inclusion in PubMed, CAS, Scopus and Google Scholar

- Research which is freely available for redistribution

Submit your manuscript at www.biomedcentral.com/submit 